\title{
Biomedical effects induced by the monocycle pulse
}

\author{
Yafang Tan ${ }^{1,2 \star}$, Hongchun Yang ${ }^{1,2}$, Jun $\mathrm{Xu}^{1,2}$ and Xiaolin Yang ${ }^{1,2}$ \\ ${ }^{1}$ School of Physical Electronics, University of Electronic Science and Technology of China, Sichuan, 610054, China. \\ ${ }^{2}$ School of Physical Electronics, University of Electronic Science and Technology of China, No.4, Section 2, North \\ Jianshe Road, Chengdu, P. R. China. \\ Accepted 18 July, 2011
}

\begin{abstract}
Based on multilayer dielectric model, for the spherical biological cell subjected to pulsed electric field (PEF), a simplified equivalent circuit was presented. According to a mathematical analysis of a monocycle electric pulse (MEP), the relationship between a given pulse duration and a center frequency was established and its main energy was concentrated on the center frequency. Thus the transmenbrane potential of external and inner membranes induced by the MEP with various durations was simply calculated by using a harmonic function with different center frequency and the equivalent circuit equations. Furthermore, the threshold electric intensities and window effect of the electroporation with different pulse duration were discussed assuming that the external and inner membranes would breakdown under certain potential values. In view of current difficulty in developing the high peak power PEF devices and the advantage of the MEP in living cell's electroporation effects, an experimental scheme was presented in which a Gaussian pulse was generated by a photoconductive semiconductor switch (PCSS) and a MEP was produced through a Blumlein transmission line (BTL).
\end{abstract}

Key words: Monocycle pulse, transmenbrane potential, electric intensity threshold, window effect, monocycle pulse generator.

\section{INTRODUCTION}

Based on the unique biomedical effects of the pulsed electric field (PEF), a new tumor treatment without drug has been proposed and widely studied in the past few years (Schoenbach et al., 2004; Cima and Mir, 2004; Butterwick et al., 2007; Schoenbach et al., 2008). Much of the work shows that PEF can cause many reversible aqueous channels (radius: 20 to $110 \mathrm{~nm}$ ) at the cell membrane under lower electric field intensity and longer pulse duration (typical parameters: $100 \mathrm{~ns}, 1 \mathrm{kV} / \mathrm{cm}$ ) (Weaver, 2000, 2003), while there is no obvious effect on the intra-membranous organelles. This physical procedure, termed as electroporation which can make cell membrane more permeable to drug molecule, has been successfully applied to tumor treatment. However, as the pulse duration decreases and voltage increases (1 $\mathrm{ns}, 10 \mathrm{kV} / \mathrm{cm}$ ), the PEF can lead to electroporation of organelle membranes, such as nucleolus, mitochondria,

*Corresponding author. E-mail: yafang-tan@163.com. Tel: +8613880520805. etc, without losing the integrity of outer membrane, and may result in apoptosis of tumor cells (Joshi et al., 2009; Schoenbach, 2007; Katsuki et al., 2007; Schoenbach et al., 2001; Stacey et al., 2003). This phenomenon is termed intracellular electromanipulation (IEM). In view of clinical application, PEF with too strong intensity or too long duration may result in irreversible injury to the surrounding healthy cells or some undesirable thermal effect and sufferings to the patient. So, it is very critical to study the window effect of PEF on biological cells in order to precisely determine the PEF parameters and get effective therapeutic effects. The mechanism and threshold conditions of IEM have been extensively researched in the past few years (Lsambent, 1998; Chalise et al., 2006; Hu et al., 2009; Gurtovenko and Vattulainen, 2009; Pucihar et al., 2009; Sel et al., 2009; Hu et al., 2005; Joshi et al., 2002; Daniels and Rubinsky, 2009). Among the models and methods, an equivalent circuit method based on the multilayer dielectric model is popular to analyze the transmenbrane potential, the electric intensity threshold and the window effect. Its major views are that the external and inner membranes are sensitively respond to different 


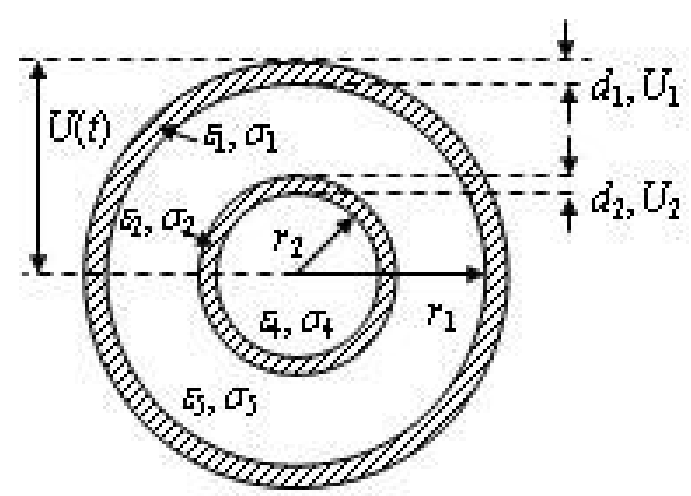

(a)

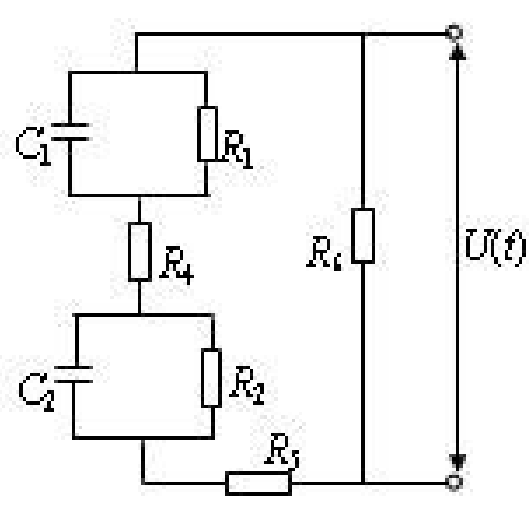

(b)

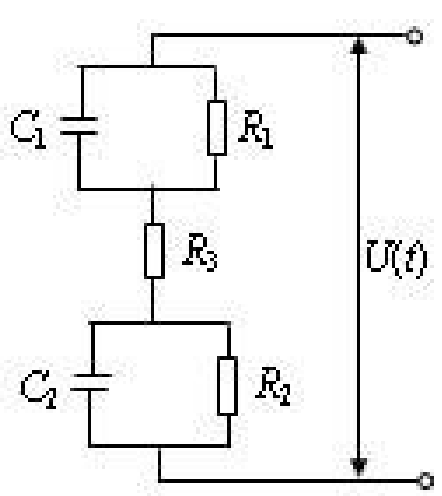

(c)

Figure 1. Multilayer dielectric model of spherical cell and its equivalent circuits with (a) multilayer dielectric model of spherical cell; (b) equivalent circuit of cell subjected to PEF and $(c)$ simplified equivalent circuit of cell subjected to PEF.

PEF parameters and were broken down as the transmembrane voltage reaches up to a given threshold voltage $(1 \mathrm{~V})$. Although the equivalent circuit analysis could not describe the micro-dynamic mechanism of the IEM and its time; frequency analysis usually could not offer the analytical results and clear physical picture of what it calculated (Yao et al., 2009), it does provide an easy and concise method to calculate the transmembrane voltage, the threshold of breakdown electric field intensity and the parameter conditions of window effect. As for a monocycle pulse, a given pulse duration is corresponded to a center frequency and its main energy is concentrated on the center frequency, thus the transmenbrane potential induced by MEP with various duration can be simply be calculated by using the harmonic function with different center frequencies employing the equivalent circuit equations. Furthermore, assuming that the external and inner membranes should be breakdown as the transmenbrane potentials are up to a certain value, respectively, the threshold electric intensity and the conditions of window effect of electroporation under different pulse durations are carried out. In view of the difficulty in developing the high peak power PEF devices and the advantage of the monocycle pulse in having effective electroporation effects, an experimental scheme is offered, in which a Gaussian pulse is generated by a photoconductive semiconductor switch (PCSS) and a MEP is outputted through a Blumlein transmission line (BTL). It is expected that electrical parameters of tumor cells can be test more precisely and biomedical experiment can be carried out more effectively by using a MEP instead of a unipolar pulse.

\section{MODEL}

As shown in Figure 1, the multilayer dielectric model and its equivalent circuit indicate that the functions of cell cytoplasm, nuclear cytoplasm and extracellular medium subjected to PEF can be regarded as a resistive load $\left(R_{4}\right.$, $R_{5}, R_{6}$ ) (Yao et al., 2009), and the functions of external and inner membrane can be regarded as a parallel connection of a resistive and a capacitive load $\left(R_{1}, C_{1}\right.$; $R_{2}, C_{2}$ ), respectively. It is easy to understand that the value of $R 6$ has no effect on the transmembrane potential of cell membrane and nuclear membrane. So the equivalent circuit can be simplified as Figure 1(c). Where $R_{3}=R_{4}+R_{5}$. According to Ohm's law and the method to calculate the capacity of parallel plate capacitor, the value of $R_{i}, C_{i}$ can be calculated as following formulae

$$
\begin{aligned}
& R_{1}=\frac{d_{1}}{\sigma_{1} s}, \quad R_{2}=\frac{d_{2}}{\sigma_{2} s}, \quad R_{3}=\frac{\left(r_{1}-r_{2}\right) \sigma_{5}+r_{2} \sigma_{4}}{\sigma_{4} \sigma_{5} s} \\
& C_{1}=\frac{\varepsilon_{0} \varepsilon_{r 1} s}{d_{1}}, C_{2}=\frac{\varepsilon_{0} \varepsilon_{r 2} s}{d_{2}}
\end{aligned}
$$

where $\sigma_{i}, d_{i}, \varepsilon_{r}, r_{i}$ are the conductivity, thickness of external and inner membranes, relative permittivity and radius of cell and nuclear, all of them are shown in Figure 1a. The parameter " $s$ " is the equivalence value of the cross-sectional area of the current. It is assumed to have the same value with each of the multilayer dielectric just because the calculation is convenient in this paper.

\section{THEORETICAL ANALYSIS}

\section{Power spectrum analysis of typical pulse}

Gaussian-type PEFs generated by conventional electronic devices can be described as a Gaussian pulse, a monocycle and a quasi-rectangular pulse shown in 


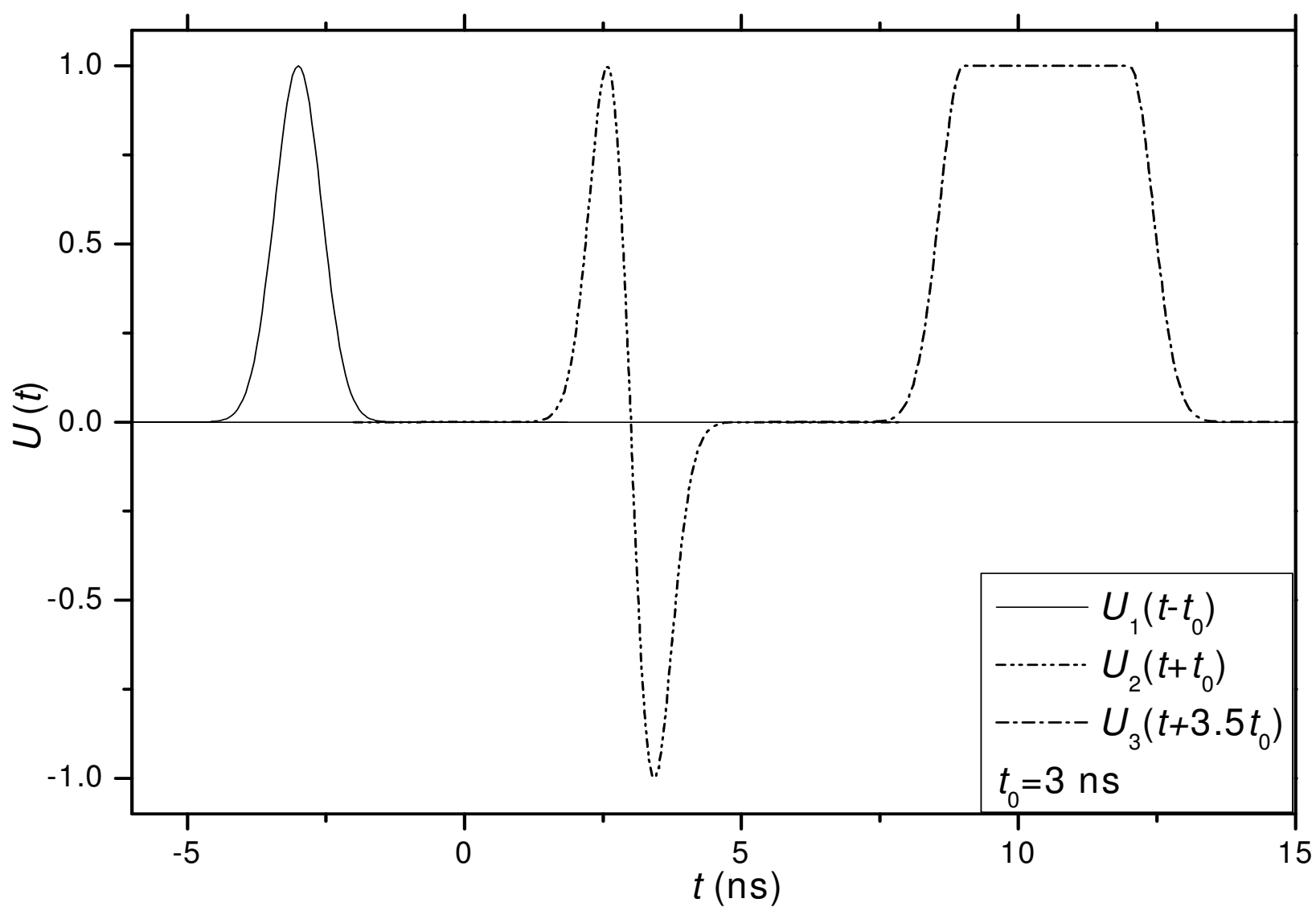

Figure 2. Gaussian-type PEFs used in IEM and electroporation effects.

Figure 2 which are usually used in IEM and electroporation effects. Gaussian function $U_{1}(t)$ with FWHM $T$ can be written as

$$
\begin{aligned}
& U_{1}(t)=U_{0} \exp \left(-\alpha t^{2}\right) \\
& \alpha=\frac{4 \ln 2}{T^{2}}
\end{aligned}
$$

Corresponding to $U_{1}(t)$, a monocycle function $U_{2}(t)$ can be written as

$$
U_{2}(t)=-\sqrt{2 \alpha} e^{1 / 2} U_{0} \cdot t \exp \left(-\alpha t^{2}\right)
$$

and a quasi-rectangular pulse can be written as

$$
U_{3}(t)= \begin{cases}U_{0} \exp \left(-\alpha t^{2}\right) & t<-\tau_{0} / 2, t>\tau_{0} / 2 \\ U_{0} & -\tau_{0} / 2<t<\tau_{0} / 2\end{cases}
$$

According to Fourier integral transform, the power spectrum of $U_{i}(t)$ can be expressed as

$$
\left\{\begin{array}{l}
P_{1}(\omega)=\frac{U_{0}^{2}}{4 \alpha \pi} e^{-\omega^{2} /(2 \alpha)} \\
P_{2}(\omega)=\frac{U_{0}^{2}}{4 \alpha \pi} \cdot \omega^{2} e^{-\omega^{2} /(2 \alpha)} \\
P_{3}(\omega)=\frac{U_{0}^{2}}{4 \pi^{2}}\left[\sqrt{\frac{\pi}{\alpha}} e^{-\omega^{2} /(4 \alpha)}+\frac{2}{\omega} \sin \frac{\tau_{0} \omega}{2}\right]^{2}
\end{array}\right.
$$

Equation (7) indicates that $P_{1}(\omega)$ and $P_{3}(\omega)$ are the same function when $\omega>>1$. The extreme condition and the maximum values of $P_{2}(\omega)$ are

$$
\left\{\begin{array}{l}
\omega_{0}=\sqrt{2 \alpha}=\frac{2 \sqrt{2 \ln 2}}{T} \\
P_{2}\left(\omega_{0}\right)=\frac{e^{-1} U_{0}^{2}}{2 \pi}=8.858 \times 10^{-2} U_{0}^{2}
\end{array}\right.
$$




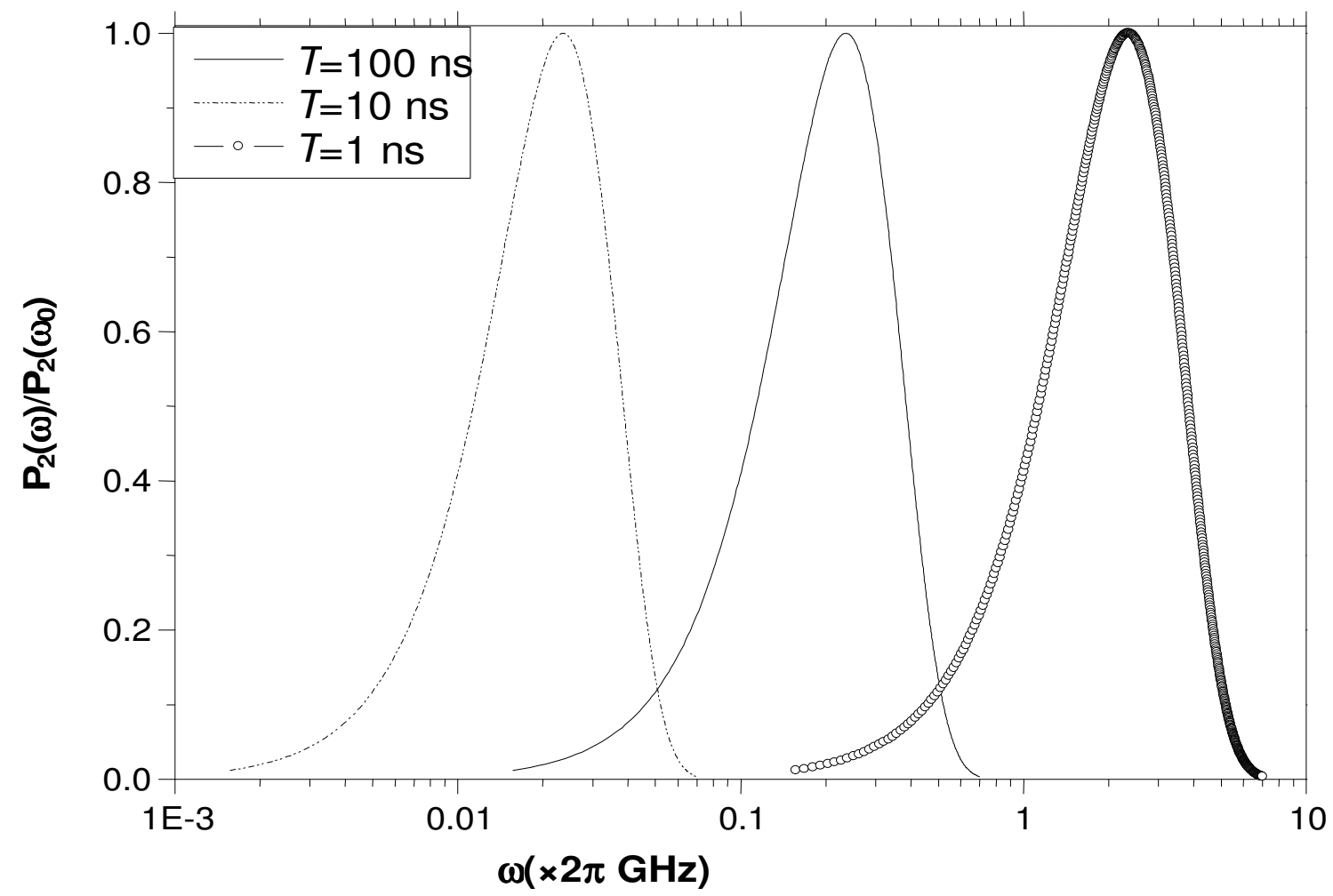

Figure 3. The power spectrum of the first-order Gaussian functions.

As $\omega=\gamma \omega_{0}$, Solving simultaneously Equations (7) and (8) gets

$\frac{P_{2}(\gamma \omega)}{P_{2}\left(\omega_{0}\right)}=\gamma^{2} \exp \left(-\gamma^{2}+1\right)$

Equation (9) indicates the value of $P_{2}(\gamma \omega) / P_{2}\left(\omega_{0}\right)$ is constant for a given $\gamma$. In combination of Equation (8), it is clear that the curve of $P_{2}(\omega)$ will moves to higher frequency as a whole as the pulse durations decrease. Figure 3 shows the curve of $P_{2}(\omega)$ under different pulse durations. It suggested that the transmembrane voltage induced by a monocycle can be regarded as the effects carried out by a harmonic sine function with a certain frequency, such as $\omega_{0}$, etc. Taking $P_{2}\left(\omega_{0}\right) / 2$ as a reference, the band of the power spectrum ranges from 0.48 to $1.634 \omega_{0}$.

\section{Transmembrane potential and window effect}

For simplicity, assuming the sine function with a center frequency $\omega_{0}$, that is

$U(t)=U_{0} \sin \left(\omega_{0} t\right)$
The circuit equations shown in Figure 1 are

$$
\left\{\begin{array}{l}
\dot{U}_{c 1}(t)=\frac{Z_{1}}{Z_{1}+Z_{2}+R_{3}} \dot{U}(t) \\
\dot{U}_{c 2}(t)=\frac{Z_{2}}{Z_{1}+Z_{2}+R_{3}} \dot{U}(t) \\
\dot{U}_{R 3}(t)=\frac{R_{3}}{Z_{1}+Z_{2}+R_{3}} \dot{U}(t)
\end{array}\right.
$$

where

$$
\left\{\begin{array}{c}
\frac{1}{Z_{1}}=\frac{1}{Z_{c 1}}+\frac{1}{Z_{R 1}}=\mathrm{i} \omega_{0} C_{1}+\frac{1}{R_{1}} \\
\frac{1}{Z_{2}}=\frac{1}{Z_{c 2}}+\frac{1}{Z_{R 2}}=\mathrm{i} \omega_{0} C_{2}+\frac{1}{R_{2}}
\end{array}\right.
$$

with $\mathrm{i}$ the imaginary unit.

Solving simultaneously Equations (11) and (12), we can obtain 


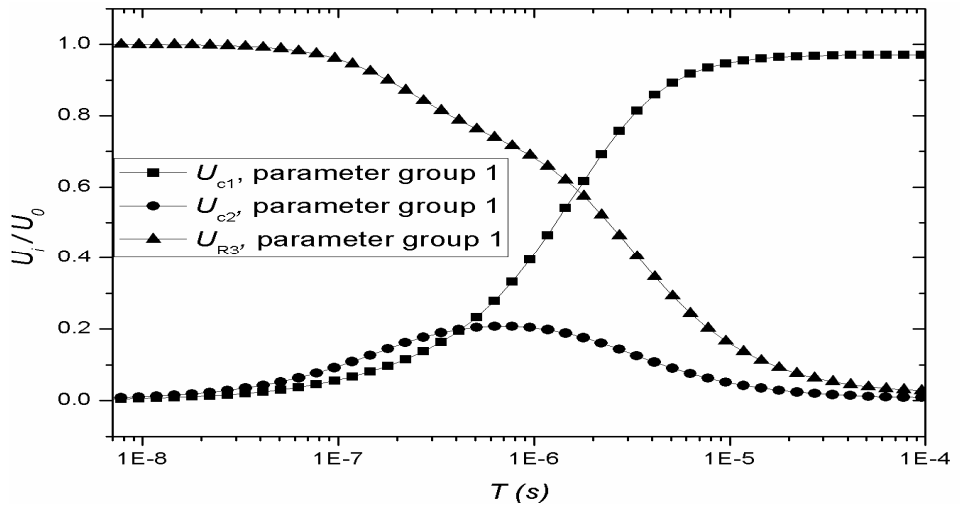

(a)

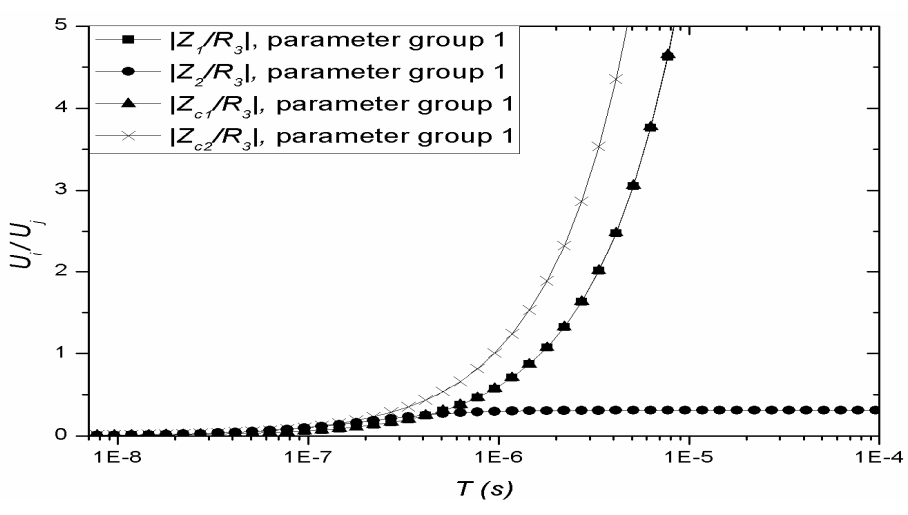

(b)

Figure 4. The transmembrane voltages vs pulse durations. (a) The transmembrane voltages changes with the pulse duration, and (b) $Z_{i} / Z_{j}$ vs pulse duration.

$$
\left\{\begin{array}{l}
\left|\frac{\dot{U}_{c 1}(t)}{\dot{U}(t)}\right|=R_{1} \sqrt{\frac{1+\left(\omega_{0} \tau_{2}\right)^{2}}{U_{k 1}^{2}+U_{k 2}^{2}}} \\
\left|\frac{\dot{U}_{c 2}(t)}{\dot{U}(t)}\right|=R_{2} \sqrt{\frac{1+\left(\omega_{0} \tau_{1}\right)^{2}}{U_{k 1}^{2}+U_{k 2}^{2}}} \\
\left|\frac{\dot{U}_{R 3}(t)}{\dot{U}(t)}\right|=R_{3} \sqrt{\frac{\left(1-\omega_{0}^{2} \tau_{1} \tau_{2}\right)^{2}+\omega_{0}^{2}\left(\tau_{1}+\tau_{2}\right)^{2}}{U_{k 1}^{2}+U_{k 2}^{2}}}
\end{array}\right.
$$

$$
\left\{\begin{array}{l}
U_{k 1}=\left(R_{1}+R_{2}\right)+R_{3}\left(1-\omega_{0}^{2} \tau_{1} \tau_{2}\right) \\
U_{k 1}=\omega_{0}\left[R_{1} \tau_{2}+R_{2} \tau_{1}+R_{3}\left(\tau_{1}+\tau_{2}\right)\right] \\
\tau_{1}=R_{1} C_{1}=\varepsilon_{0} \varepsilon_{r 1} / \sigma_{1} \\
\tau_{2}=R_{2} C_{2}=\varepsilon_{0} \varepsilon_{r 2} / \sigma_{2}
\end{array}\right.
$$

where The transmembrane potential of the external and inner membranes calculated by Equations (1), (2), (8), (13) and (14) are shown in Figure 4, where cell parameters used in calculation are shown in Table $1 \mathrm{Hu}$ et al., 2005; Yao et al., 2009). The calculations indicate the external and inner transmembrane voltages are closely related to the PEF durations. If the pulse duration is longer than several microseconds, Figure $4 a$ demonstrates that the bias voltage is mainly applied to the external membrane. Figure $4 \mathrm{~b}$ shows $\left|Z_{1} / R_{3}\right| \approx\left|Z_{c 1} / R_{3}\right| \approx R_{1} / R_{3}>>1$ and $\left|Z_{2} / R_{3}\right| \approx R_{2} / R_{3}$, so the value of $U_{c 1}$ is determined by the ratio of $R_{1}$ and $R_{1}+R_{2}+$ $R_{3}$. That is to say the transmembrane potentials are mainly determined by the conductivity parameters of multilayer dielectric. On the other hand, if the pulse duration is shorter than dozen nanoseconds or sub nanosecond, Figure $4 \mathrm{a}$ shows that the bias voltage is mainly applied to $R_{3}$. Figure $4 \mathrm{~b}$ shows $\left|Z_{1} / R_{3}\right| \approx\left|Z_{c 1} / R_{3}\right|$ $<<1$ and $\left|Z_{2} / R_{3}\right| \approx\left|Z_{c 2} / R_{3}\right|<<1$, so the value of $U_{R 3}$ is determined by the ratio of $R_{1}$ and $Z_{c 1}+Z_{c 1}+R_{3} \approx R_{3}$. In other words, the relative permittivity parameters of external and inner membranes mainly determine the pulse duration by which the bias voltage is only applied on the $R_{3}$. However, if the pulse duration is between dozen nanoseconds and a few micro-seconds, Figure 4a denotes the bias voltage is mainly applied to the inner membrane and $R_{3}$. Figure $4 \mathrm{~b}$ shows $\left|Z_{1} / R_{3}\right| \approx\left|Z_{c 1} / R_{3}\right|$ decreasing rapidly and $\left|Z_{2} / R_{3}\right| \approx\left|Z_{c 2} / R_{3}\right|$. That is to say only when the pulse duration takes certain values, it has the value of $Z_{c 1}$ or $Z_{c 2}$ equivalent to $R_{3}$, the potential of inner membrane can be up to its maximum values. This phenomenon is termed the window effect. Figure 4 shows the pulse duration corresponding to window effect may be written as

$$
Z_{c 1} \leq R_{3} \leq Z_{c 2}
$$

Solving simultaneous Equations (1), (2) and (8) gets

$$
\frac{k_{1} \varepsilon_{r 2}}{d_{2}} \leq T \leq \frac{k_{1} \varepsilon_{r 1}}{d_{1}}
$$

where

$$
k_{1}=\frac{2 \sqrt{2 \ln 2} \varepsilon_{0}\left[\sigma_{5}\left(r_{1}-r_{2}\right)+\sigma_{4} r_{2}\right]}{\sigma_{4} \sigma_{5}}
$$

As for a certain tumor cell, Equations (16) and (17) show how the window effect is affected by the parameters and pulse duration. It is easy to understand the importance 
Table 1. Simulating parameters of cell.

\begin{tabular}{llcc}
\hline Parameter & Cell component & Group 1 & Group 2 \\
\hline & Cell membrane $\sigma_{1}$ & $5.3 \times 10^{-6}$ & $1 \times 10^{-5}$ \\
Conductivity $(\mathrm{S} / \mathrm{m})$ & Cell cytoplasm $\sigma_{4}$ & 0.13 & 0.5 \\
& Nuclear membrane $\sigma_{2}$ & $4.3 \times 10^{-3}$ & $1 \times 10^{-3}$ \\
& Nuclear cytoplasm $\sigma_{5}$ & 0.18 & 1 \\
& & & \\
& Cell membrane $\varepsilon_{r 1}$ & 7 & 10 \\
Relative permittivity & Cell cytoplasm $\varepsilon_{r 4}$ & 60 & 80 \\
& Nuclear membrane $\varepsilon_{r 2}$ & 22.8 & 10 \\
& Nuclear cytoplasm $\varepsilon_{r 5}$ & 120 & 80 \\
& & & \\
& Cell radius $r_{1}$ & 5.12 & 10 \\
& Cell membrane thickness $d_{1}$ & 0.007 & 0.007 \\
Geometry parameter $(\mu \mathrm{m})$ & Nuclear radius $r_{2}$ & 4.4 & 5 \\
& Nuclear membrane thickness $d_{2}$ & 0.04 & 0.04 \\
\hline
\end{tabular}

that different waveform should be applied to different tumor cells.

\section{The threshold parameters of biomedical effects}

Assuming that the external and inner membranes should breakdown when the transmembrane voltages are up to the given value $U_{k 1}$ and $U_{k 2}$, respectively, the electric filed intensity threshold of $E_{k 1}$ and $E_{k 2}$ under a PEF with pulse duration $T$ can be carried out based on the calculations of the transmembrane voltage as follows indicates

$$
E_{k 1} \geq \frac{U_{k 1}}{r_{1}} \frac{U_{0}}{U_{c 1}} \text { and } \quad E_{k 2} \geq \frac{U_{k 2}}{r_{2}} \frac{U_{0}}{U_{c 2}}
$$

Figure 5 shows the electric field intensity threshold in different pulse durations under the conditions of $U_{k 1}=U_{k 2}=1 \mathrm{~V}$. The calculations indicate that: (1) If the pulse duration is longer than several microseconds, the external membrane should be breakdown under a lower field intensity about $1 \mathrm{kV} / \mathrm{cm}$ while the inner membrane needs $10 \mathrm{kV} / \mathrm{cm}$.

On the other hand, If the pulse duration is shorter than dozens of nanoseconds, both of the external and inner membrane should be breakdown only when the field intensity is great than $10 \mathrm{kV} / \mathrm{cm}$. However, if the pulse duration is determined by Equation (16), the inner membrane should be breakdown under a lower field intensity about $10^{\circ} \mathrm{kV} / \mathrm{cm}$ while the external membrane needs $10^{1} \mathrm{kV} / \mathrm{cm}$. (2) It is easy to explain that the external and the inner membranes should be breakdown at the same time under the conditions that the filed intensity is about $10^{\circ} \mathrm{kV} / \mathrm{cm}$ and the pulse duration is about $10^{2} \mathrm{~ns}-$
$10^{1} \mu \mathrm{s}$, because the transmembrane voltages applied to the two membranes are all larger than the threshold intensity as shown in Figure 5. (3) Window effect is sensitive to the cell's parameters. Figure 5 shows the differences between two group cell's parameters. It means that the pulse duration and the waveform of PEF should be precisely designed according to its main energy, which is concentrated on the area that can make sure the window effect occurred effectively.

\section{EXPERIMENTAL SCHEME}

There are three reasons to offer the following experimental scheme. (1) The fact that the IEM effects lead to the cell's apoptosis but harmless to the normal cells and tissues. It suggests that the generator produces high voltage nanosecond electric pulses, which can induce IEM effect effectively, is one of the keys for the IEM studies. (2) That experiments affirm that the monocycle PEF can induce more effective electroporation than the unipolar PEF. It is necessary to pay more attention to study the IEM effects using monocycle PEF. (3) The calculations shown in Figures 4 and 5 denote that the theoretical results are closely related to the accuracy test of the cell's electrical parameters while these parameters are varied significantly from different references.

In order to get more accurate parameters' value and IEM effects, the advantages of the monocycle PEF such as a certain bandwidth, concentrate energy, etc. should be taken into account.

\section{A biomedical effect of unipolar and bipolar PEF}

Vernier et al. 2006) reported an experiment which showed 


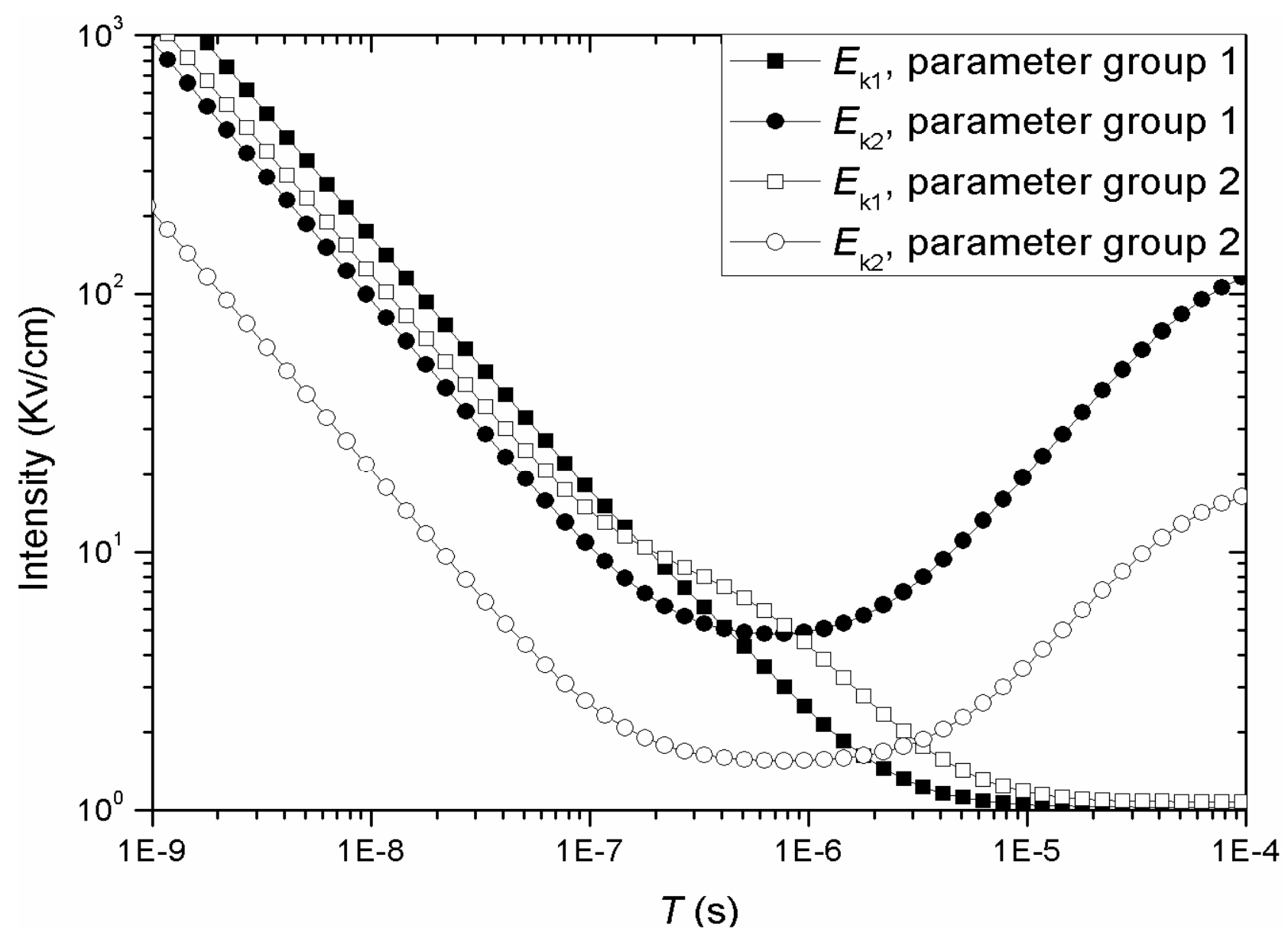

Figure 5. The threshold relationship between the breakdown filed intensity and the pulse durations.

the difference between unipolar and biopolar PEFs. Bipolar pulses redistribute phospholipids at both the anode and cathode poles, consistent with migration of the anionic PS head group in the transmembrane field, while unipolar pulses perturb membrane phospholipid order only at the anode side of the cell, tracked by FM1-43 fluorescence. Although, the equivalent circuit model could not describe the micro- dynamic mechanism concisely now, but it is sure that the bipolar PEF can induce more effective electroporation.

\section{The method of a monocycle PEF production}

In Figure 6, a right-traveling Gaussian pulse is generated by a PCSS illuminated by a laser pulse, the right-traveling pulse is outputted to the load after a time of $l_{1} /\left(c \varepsilon_{r}^{1 / 2}\right)$ and is coupled into the microstrip with length $l_{2}$ at the same time. The coupling pulse is reflected with half-wave loss when it gets to the right end point and become a lefttraveling pulse. Then it is in superposition with the righttraveling pulse at the output point and formed a monocycle pulse. It is easy to prove that the bipolar pulse's waveform can be expressed as a first-order Gaussian function with an identical maximum waveform to the Gaussian if the length of $l_{1}$ and $l_{2}$ are appropriately matched. The superposition waveform can be expressed as:

$$
U_{1}(t)=U_{0}\left\{\exp \left[-\alpha\left(t-t_{0}\right)^{2}\right]-\exp \left[-\alpha\left(t+t_{0}\right)^{2}\right]\right\}
$$

where $t_{0}=l_{2} /\left(c \varepsilon_{r}^{1 / 2}\right)$

Let

$t_{0}=\frac{1}{\sqrt{\alpha}}$

Solving simultaneous Equations (19) and (20) gets

$U_{1}\left(t_{0}\right)=U_{0}\left[1-\exp \left(-4 \alpha t_{0}^{2}\right)\right] \approx U_{0}$ 


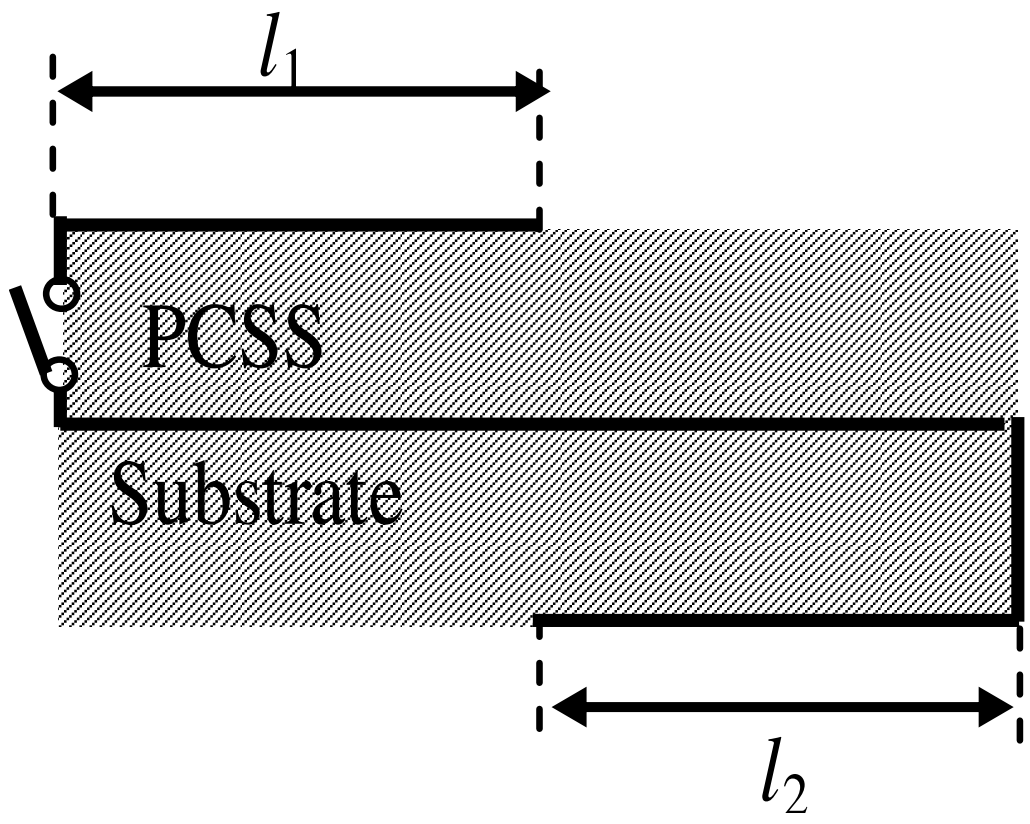

Figure 6. The first-order Gaussian pulse generated by a PCSS and a BTL.

Equation (19) indicates that the maximums of Equations (19) and (21) are equal. Assuming that Equation (19) can be expressed by a first-order Gaussian as

$$
U_{2}^{\prime}(t)=2 \gamma_{3} U_{0} \alpha^{\prime} t \cdot \exp \left(-\alpha^{\prime} t^{2}\right)
$$

The time $T_{1}$ between the two extreme point decided by Equation (22) is

$$
T_{1}=\frac{T^{\prime}}{\sqrt{2 \ln 2}}=\frac{1}{\sqrt{\alpha^{\prime}}}
$$

The condition should be satisfied in order to express Equation (19) by (22)

$$
T_{1}=2 t_{0} \Rightarrow \alpha^{\prime}=\frac{\alpha}{2}
$$

Equations (20) and 23) are used in the deduction process. Solving simultaneously Equations (22) to (24), and taking into account $U_{2}\left(t_{0}\right)=U_{0}$ while $t= \pm t_{0}$, then get

$$
U_{2}^{\prime}(t)=e^{1 / 2} \sqrt{\alpha} U_{0} t \cdot \exp \left(-\frac{\alpha}{2} t^{2}\right)
$$

Figure 7 plots the curves of Equations (20) and (23) and suggests that Equation (19) can be well expressed by Equation (25). It indicates that the first-order Gaussian pulse can be obtained with a Gaussian pulse by using a BTL if the length of $I_{1}$ and $I_{2}$ are appropriately matched.

\section{Design of the monocycle PEF generator device}

The scheme of the monocycle PEF generator is shown in Figure 8. An optical-electric synchronizer controls the time delay between the pulsed power supply and the miniaturized laser diode illumination system in order to have the PCSS in its on-state as soon as the BTL has been charged. The Gaussian pulse generated by the PCSS is transformed to a monocycle through the BTL. In the past few years, a pulsed power supply with $15 \mathrm{kV}$ maximum output voltage, $0.44 \mathrm{~kg}$ weight, $10 \times 8 \times 6 \mathrm{~cm}^{3}$ volume and maximum $1 \mathrm{kHz}$ repeat frequency rate (RFR) has been developed based on the Flyback transformer technique. An optical-electric synchronizer with 10 ns delay resolution and $1 \mathrm{~ms}$ delay range was reported (Zeng et al., 2008).

At the same time, a $3 \mathrm{~mm}$ gap opposed-contact GaAs PCSS integrated with the BTL, by which a Gaussian pulse with $15 \mathrm{kV}$ maximum voltage, 1 to $10 \mathrm{~ns}$ pulse duration, $1 \mathrm{kHz}$ RFR can be output, was also reported (Zeng et al., 2008; Mar et al., 2000). So it is possible to develop a monocycle generator by using the techniques as denoted previously, and it would be useful for the IEM effects study and the electrical parameters test of the tumor cells.

\section{CONCLUSION}

In view of the advantages of the MEP, such as certain bandwidth and concentrate energy, a new simple analytical method, by which the function of fed MEP is instead by an harmonic function with a certain center 


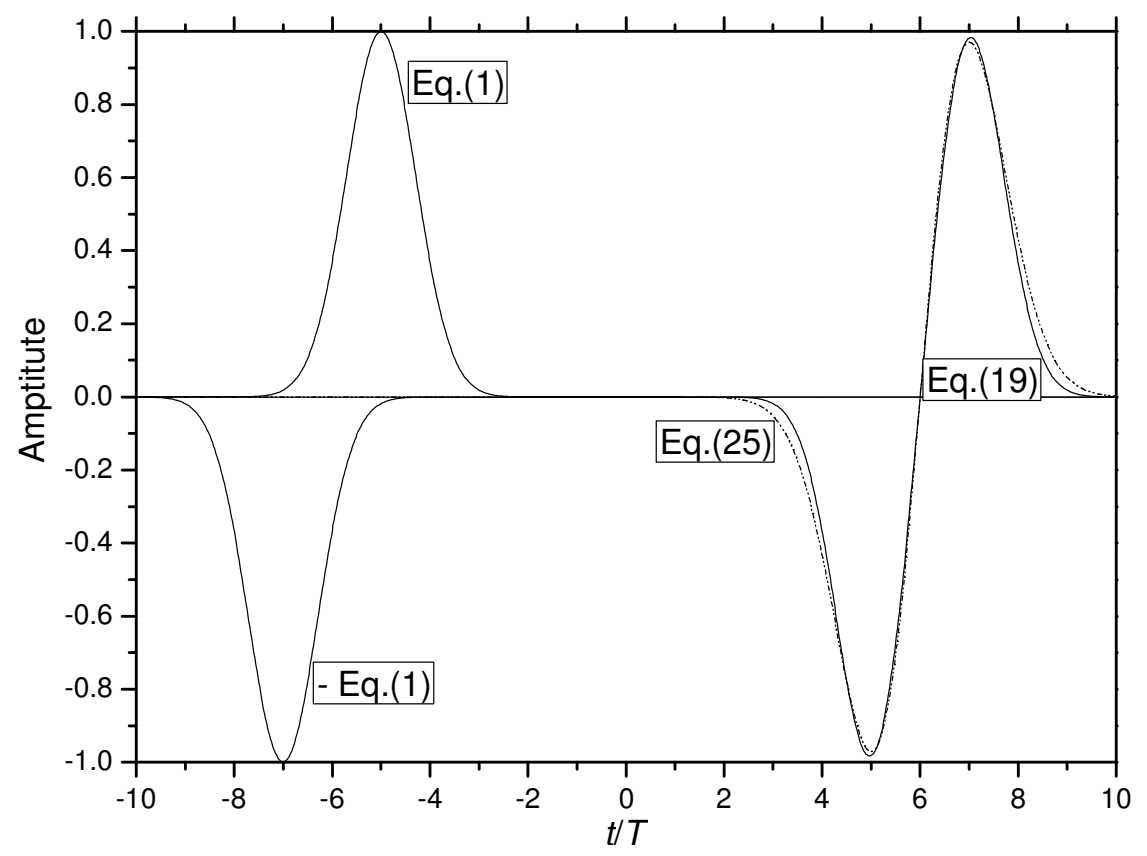

Figure 7. The curves of Equations (19) and (25).

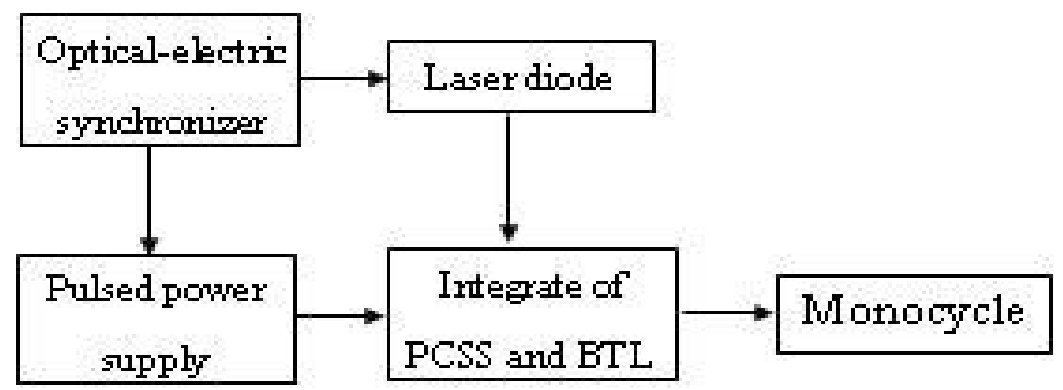

Figure 8. The schematic diagram of the first-order Gaussian PEF generator.

frequency, is offered. It can describe the window effect, explain many phenomena of IEM and electroporation concisely in contrast to the complexion of the timefrequency analysis. It suggests that all these phenomena are caused by the different actions of resistance and capacity induced by cell membranes, cell cytoplasm and nuclear cytoplasm under different PEF's parameters, and declares that the biomedical effects are not only relay on the parameters of cell and PEF, but also sensitive to the waveform of PEF. As for the PEFs with the same pulse duration or energy, different waveform means different power spectrum distribution. It is the spectrum distribution which decides whether the window effect would occur or the efficiency of biomedical effect to a certain tumour cell. Because experiments has denoted that the MEP can induce more effective electroporation and other conclusions induced by this paper, Furthermore, there is no more difficulty to produce a MEP generator than a unipolar's. It is reasonable to pay more attention to study the biomedical effects induced by different waveform of the PEF.

\section{ACKNOWLEDGEMENT}

The present work is supported by National Natural Science Foundation of China (Grant No. 10804016).

\section{REFERENCES}

Butterwick A, Vankov A, Freyvert P, Palanker D (2007). Tissue damage by pulsed electrical stimulation. IEEE T. Biomed. Eng., 54: 22612267.

Chalise PR, Perni S, Shama G, Novac BM, Smith IR, Kong MG (2006). Lethality mechanisms in Escherichia coli induced by intense submicrosecond electrical pulses. Appl. Phys. Lett., 89: 153902. 
Cima LF, Mir LM (2004). Macroscopic characterization of cell electroporation in biological tissue based on electrical measurements. Appl. Phys. Lett., 85: 4520-4522.

Daniels C, Rubinsky B (2009). Electrical field and temperature model of nonthermal irreversible electroporation in heterogeneous tissues. J. Biomech. Eng., 131: 071006.

Gurtovenko AA, Vattulainen I (2009). Calculation of the electrostatic potential of lipid bilayers from molecular dynamics simulations: Methodological issues. J. Chem. Phys., 130: 215107.

$\mathrm{Hu}$ Q, Joshi RP, Schoenbach KH (2005). Simulations of nanopore formation and phosphatidylserine externalization in lipid membranes subjected to a high-intensity, ultrashort electric pulse. Phys. Rev. E., 72: 031902.

Hu Q, Joshi RP, Beskok A (2009). Model study of electroporation effects on the dielectrophoretic response of spheroidal cells. J. Appl. Phys., 106: 024701.

Joshi RP, Hu Q, Schoenbach KH, Hjalmarson HP (2002). Theoretical predictions of electromechanical deformation of cells subjected to high voltages for membrane electroporation. Phys. Rev., E. 65: 021913.

Joshi RP, Song J, Schoenbach KH, Sridhara V (2009). Aspects of lipid membrane bio-responses to subnanosecond, ultrahigh voltage pulsing. IEEE T. Dielect. El. In., 16: 1243-1250.

Katsuki S, Nomura N, Koga H, Akiyama H, Uchida I, Abe S-I (2007). Biological effects of narrow band pulsed electric fields. IEEE T. Dielect. El. In., 14: 663-667.

Lsambent H (1998). Understanding the electroporation of cells and artificial bilayer membranes. Phys. Rev. Lett., 80: 3404-3407.

Mar A, Louberiel GM, Zutarvern FJ, O'Malley MW, Helgeson WD, Brown DJ, Hjalmarson HP, Baca AG, Thornton RL, Donaldson RD (2000). Doped contacts for high-longevity optically activated, high gain GaAs photoconductive semiconductor switches. IEEE T. Plasma. Sci., 28: 1507-1511.

Pucihar G, Miklavcic D, Kotnik T (2009). A time-dependent numerical model of transmembrane voltage inducement and electroporation of irregularly shaped cells. IEEE T. Biomed. Eng., 56: 1491-1450.
Schoenbach KH, Beebe SJ, Buescher ES (2001). Intracellular effect of ultrashort electrical pulses. Bioelectromagnetics, 22: 440-448.

Schoenbach KH, Joshi RP, Kolb JF, Chen NY, Stacey M, Blackmore PF, Buescher ES, Beebe SJ (2004). Ultrashort electrical pulse open a new gate into Biological cells. P. IEEE., 92:1122-1137

Schoenbach KH, Hargrave B, Joshi RP, Kolb JF, Nuccitelli R, Osgood C, Pakhomov A, Stacey M, Swanson RJ, White JA, Xiao S, Zhang J, Beebe SJ, Blackmore PF, Buescher ES (2007). Bioelectric effects of intense nanosecond pulses. IEEE T. Dielect. El. In. 14: 1088-1109.

Schoenbach KH, Xiao S, Joshi RP, Camp TJ, Tammo H, Kolb JF, Beebe SJ (2008). The effect of intense subnanosecond electrical pulses on biological cells. IEEE T. Plasma. Sci., 36: 414-421.

Sel D, Lebar AM, Miklavcic D (2009). Feasibility of employing modelbased optimization of pulse amplitude and electrode distance for effective tumor electropermeabilization. IEEE T. Biomed. Eng., 54: 773-782.

Stacey M, Stickley J, Fox P, Statler V, Schoenback K, Beebe SJ, Buescher S (2003). Differential effects in cells exposed to ultra-short, high intensity electric fields: cell survival, DNA damage, and cell cycle analysis. Mutat. Res., 542: 65-75.

Vernier PT, Sun YH, Gundersen MA (2006). Nanoelectropulse-driven membrane perturbation and smallmolecule permeabilization.BMC Cell. Biol., 7: 37.

Weaver JC (2000). Electroporation of cells and tissues. IEEE T. Plasma Sci., 28: 24-33.

Weaver JC (2003). Electroporation of biological membranes from multicellular to nano scales. IEEE T. Dielect. El. In., 10: 754-768.

Yao CG, Hu XQ, Mi Y, Li CX, Sun CX (2009). Window effect of pulsed electric field on biological cells. IEEE T. Dielect. El. In., 16: 12591266.

Zeng G, Yang XL, Yang HC (2008). Design of full digital time-lapse trigger. Exp. Sci. Tech., 6: 4-5. 